\title{
Early Keratinocyte Differentiation on Micropillar Interfaces
}

\author{
Thorsten Steinberg, ${ }^{*, \dagger}$ Simon Schulz,, Joachim P. Spatz,, Niels Grabe, ${ }^{\S}$ \\ Eva Mussig, ${ }^{\dagger}$ Annette Kohl, ${ }^{\dagger}$ Gerda Komposch, ${ }^{\dagger}$ and Pascal Tomakidi ${ }^{\dagger}$ \\ Department of Orthodontics and Dentofacial Orthopedics, Dental School, University of \\ Heidelberg, Im Neuenheimer Feld 400, D-69120 Heidelberg, and Biophysical \\ Chemistry, University of Heidelberg, Im Neuenheimer Feld 253, D-69120 Heidelberg
}

Received September 27, 2006; Revised Manuscript Received December 7, 2006

\begin{abstract}
We employed topographical patterning to analyze early keratinocyte differentiation on top of microfabricated pillar arrays. Fibronectin immobilized on pillar "heads" yielded a nucleus-associated granular keratin 1 (K1) pattern in immortalized human gingival keratinocytes (IHGK) at pillar interspaces of $14 \mu \mathrm{m}$. Decreasing distances of 11 and $8 \mu \mathrm{m}$ revealed cytoplasmic extension of the early differentiation marker $\mathrm{K} 1$ on poly(dimethylsiloxane) (PDMS) pillars. The most extensive cytoplasmic K1 protein distribution noted at the smallest pillar scale coincided with higher ratios of K1 mRNA gene transcription. These experiments suggest that early keratinocyte differentiation was governed by the topographical characteristics of the pillar pattern. Moreover, they form the basis to study cell functions such as differentiation in a defined topologically structured environment.
\end{abstract}

The discovery of generating biofunctionalized biomimetic model surfaces has opened a new dimension in nano- and microtechnology. Particularly, the combination of these material technologies with the life sciences will lead to the elucidation of fundamental requirements needed for the cells to properly exert tissue-specific functions. On the nanoscale, integrin recognition sequences used for biofunctionalization of gold dots have shown that adhesion of osteoblasts occurs in a distance-dependent manner. ${ }^{1}$ On the microscale, a new generation of model surfaces based on poly(dimethylsiloxane) (PDMS) pillars has allowed for studying the adaptation of the cytoskeleton to external mechanical and biochemical stimulations. ${ }^{2}$ Moreover, biofunctionalization of discretized pillar surfaces has been employed for cell attachment. ${ }^{3}$ These elastomeric model surfaces have a tremendous advantage in that they render micromechanical sensors for the measurement of mechanical interactions between cells and their extracellular environment. ${ }^{3,4}$ The present study addresses for the first time a further step in the versatility of these elastomeric model surfaces and includes their use as tools to study decisive cell functions in human keratinocytes.

In addition to proliferation, differentiation is a key cell function of keratinocytes to achieve and maintain epithelial

* Corresponding author. E-Mail: thorsten.steinberg@med.uni-heidelberg.de.

Department of Orthodontics and Dentofacial Orthopedics, Dental School, University of Heidelberg.

$\doteqdot$ Biophysical Chemistry, University of Heidelberg, and Max-PlanckInstitute for Metals Research, Heissenbergstrasse 3, D-70569 Stuttgart, Germany.

$\S$ Department of Medical Informatics University of Heidelberg, Im Neuenheimer Feld 400, D-69120 Heidelberg, Germany. tissue integrity, i.e., tissue homeostasis. ${ }^{5}$ In squamous epithelia, differentiation occurs during keratinocyte migration from the basal to the uppermost cell layers, including morphological and biochemical changes. The biochemical changes are reflected by the synthesis of certain molecules indicating early and terminal stages of differentiation. While involucrin $^{6}$ and filaggrin ${ }^{7}$ are markers of terminal differentiation, keratins 1 and 10 characterize early differentiation in keratinized epithelia. ${ }^{8}$ The keratin gene family comprises 30 members, and with respect to differentiation, expression of $\mathrm{K} 1$ precedes that of K10. ${ }^{9}$ For epithelial homeostasis, the adhesion of keratinocytes to extracellular matrix molecules is indispensable. Parts of the epithelial keratinocyte matrix form the epithelial basement membrane, while matrix constituents such as fibronectin $(\mathrm{FN})$ are ubiquitously found in epithelial and connective tissue. $\mathrm{FN}$ is a high-molecularweight glycoprotein, and three types have been identified so far. ${ }^{10} \mathrm{FN}$ promotes cell adhesion and affects cell morphology, migration differentiation, and cytoskeletal organization. ${ }^{11,12}$ Concerning epithelial keratinocytes, $\mathrm{FN}$ is a pivotal matrix molecule in wound healing ${ }^{13}$ and has also been described to be exclusively involved in terminal differentiation. ${ }^{14}$ Moreover, FNs are found to be associated with inflammation, including that of oral tissues. ${ }^{15}$

In contrast to our approach investigating keratinocyte differentiation in dependency of a defined microstructured environment, previous studies explored differentiation by varying the medium concentration of calcium, known as one of the major modulators of keratinocyte differentiation. In vitro experiments have shown that keratinocytes maintain 

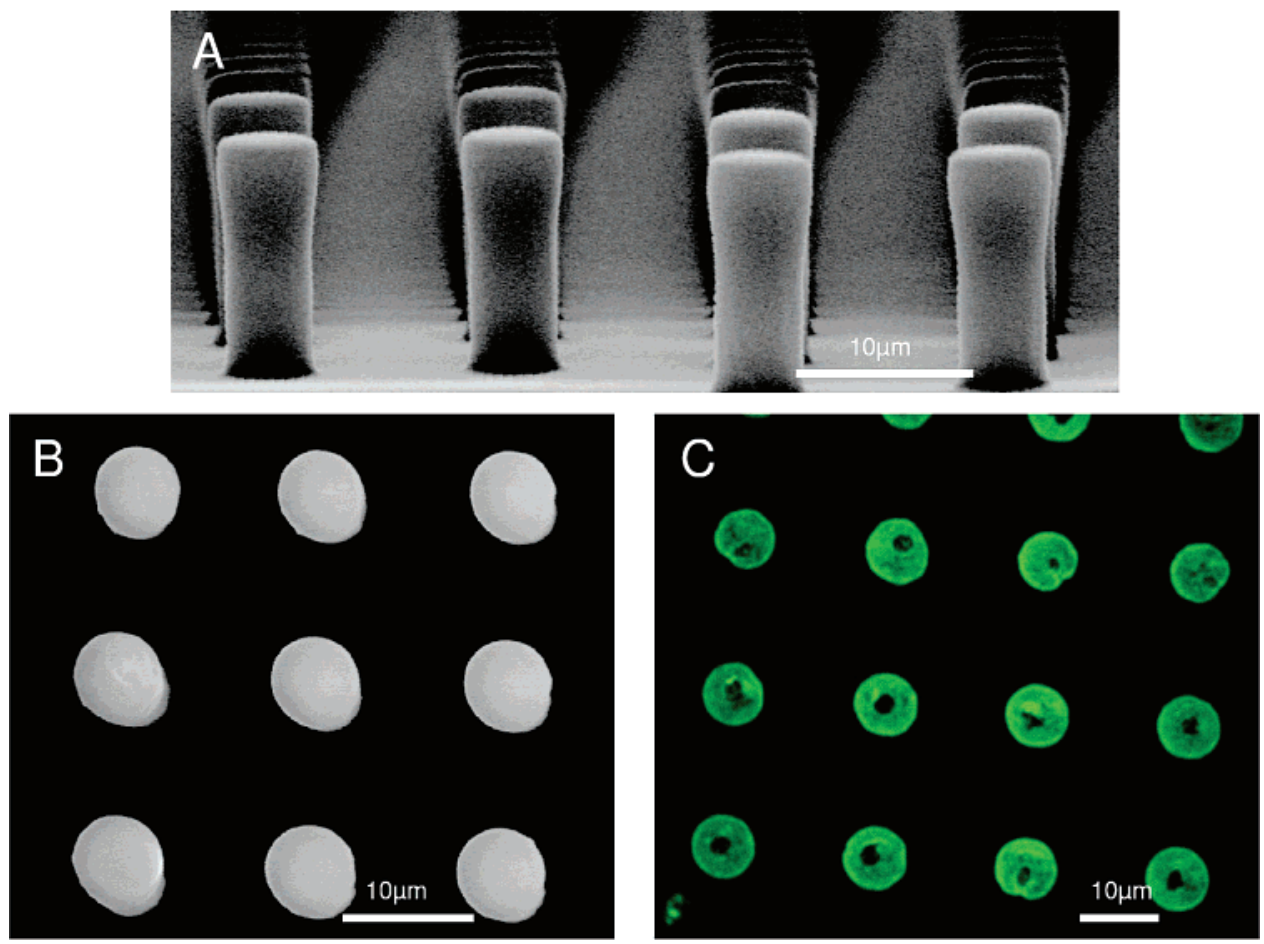

Figure 1. SEM images of (A) single PDMS micropillars from a lateral perspective illustrating the morphology of single pillars with a height of $15 \mu \mathrm{m}$ and a diameter of $5 \mu \mathrm{m}$. (B) A pillar field from top view displaying constant pillar distances of $8 \mu \mathrm{m}$ and a pillar head diameter of $5 \mu \mathrm{m}$. (C) Indirect immunofluorescence (IIF) indicates FN biofunctionalized pillar "heads" in an array of $11 \mu \mathrm{m}$ pillar top distances. The scale bars correspond to $10 \mu \mathrm{m}$.

their proliferative capacity when calcium levels in the culture medium are kept below $0.1 \mathrm{mM}$ and that differentiation ensues with calcium levels of $0.1 \mathrm{mM}$ or higher. ${ }^{16}$ Moreover, differentiation-specific genes including keratins K1 and K10 can be induced in cultured keratinocytes by raising the level of calcium in the medium. ${ }^{17}$ In the in vivo situation, rising calcium concentrations have been measured from the basal to the granular layers of the squamous epidermis epithelium, ${ }^{18}$ which similarly to the oral gingival epithelium accounts for the keratinized epithelia. ${ }^{19}$

Here, we performed studies on cell functions, such as early keratinocyte differentiation by using FN-coated pillars, arranged in patterns of defined microscaled distances. In this context, our interest focused on cell adhesion on pillar model surfaces and the modulation of early keratinocyte differentiation depending on distinct pillar distances. Because of its role in early cell differentiation of keratinized epithelia, K1 was first analyzed on the protein level by indirect immunofluorescence. In this context, it should be noted that the biofunction of intermediate filaments such as K1 is not only determined by their expression but also by their dissemination in the cell's cytoplasm. While immortalized human gingival keratinocytes (IHGK) generally exhibited a proper viability and cell adhesion on the FN-coated pillars, K1 expression indicated a mostly perinuclear protein distribution on largedistance pillar arrays. On the other hand, declining pillar distances revealed an increasing $\mathrm{K} 1$ dissemination in the cells cytoplasm. For declining pillar arrays, cytoplasmic K1 protein extension coincided with elevated levels of mRNA gene transcription. These findings indicate that pivotal cell functions such as early keratinocyte differentiation, are modulated by a defined microstructured environment.

The PDMS pillars have been generated by photolithography with the help of newly fabricated molds with holes of defined height and diameter. For this purpose, the molds were emulsified with a homogeneous thick layer of photoreactive polymer. ${ }^{20}$ One key to our experiments was to facilitate adhesion of IHGKs on the pillars. Because of its role in healing epithelial wounds and its use as a cell adhesion supporting molecule for many cell types, ${ }^{21,22}$ we have chosen the ECM molecule FN for pillar biofunctionalization. To ensure keratinocyte adhesion selectively occurring on the pillar tops, FN should be configured on the PDMS pillar tops only. In this context, it has been recently reported that FN-biofunctionalized micropillars have been employed to successfully support adhesion of mouse myoblastic cells. ${ }^{23}$ This is different from experiments under conventional in vitro cell culture conditions when FN was homogeneously dispersed on the petri culture dish. ${ }^{24}$ Although adhesion of cells was favored by such homogeneous coating, this FN configuration had the disadvantage that cellular events could not be assigned to a distinct extracellular environment.

In our studies, we used PDMS pillars designed in arrays with a pillar head diameter of $5 \mu \mathrm{m}$, a height of $15 \mu \mathrm{m}$, and different pillar interspaces, namely 14,11 , and $8 \mu \mathrm{m}$. Figure 1A illustrates a front view of a microfabricated pillar array documented by scanning electron microscopy (SEM). PDMS is a hydrophobic material, and the PDMS pillar microstructure leads to water enclosure, also known as the so-called lotus effect. ${ }^{25}$ This is important to note because a water drop 
deposited on the pillar arrays only wets the pillar heads but not the interspaced region. To visualize successful biofunctionalization of the pillar tops, indirect immunofluorescence (IIF) was performed. While the scanning electron micrograph given in Figure 1B shows the pillar tops from the top view, Figure 1C depicts the FN-coated tops. IIF demarcates FN due to coupling of a specific primary antibody and detection of the antigen-specific primary antibody by a secondary antibody coupled with a green fluorochrome (for details, see Supporting Information). Because the green fluorescence is exclusively seen on the pillar heads and the interspaced regions were devoid of fluorescence, it can be assumed that seeded cells are capable of adhering only on the pillar tops.

Thus, the FN coat is a first step in the direction of creating biofunctional and/or more biomimetic model surfaces, which represent a defined microenvironment for cells. Such biomimetic and micro-/nanostructured interfaces became more and more attractive because tissue regeneration requires the knowledge of optimal molecular conditions, which cells of certain tissues need for optimal fulfillment of their biological function. Among these conditions, the extracellular environment appears to be an important issue because it comprises matrix molecules, facilitating cell adhesion. This adhesion is mandatory for all cells of solid tissues for entering a cell cycle and later synthesis of cell- and tissue-specific differentiation products. ${ }^{26}$

To study adhesion and early differentiation of IHGKs on FN-biofunctionalized pillar arrays with different pillar distances, the keratinocytes were seeded in a constant cell number of $1 \times 10^{5} \mathrm{IHGK} / \mathrm{cm}^{2}$ on the micropillar surfaces. Establishment of IHGKs has been recently described, and the cultivation has been done in the presence of a low calcium medium to avoid calcium-dependent induction of early keratinocyte differentiation (corresponding methods are described in detail in the Supporting Information). On the basis of experiences obtained from conventional keratinocyte monolayer cultures established in routinely used plastic culture dishes, proper cell adhesion can be evaluated upon overnight $(24 \mathrm{~h})$ cultivation. Therefore, we have also estimated putative cell adhesion of IHGK on FN-coated pillar tops after the above-mentioned cultivation period. Scanning electron micrographs revealed successful and satisfactory keratinocyte adhesion to the biofunctionalized pillar tops. In comparison with the cell spreading observed for single cells on glass as a substrate (Figure 2A), microfabricated pillar arrays illustrate also fully spread single cells occupying six up to eight pillars (Figure 2C). Well-established cellular adhesion structures originating from IHGK are shown in the high-power micrograph provided by Figure 2C1 (adhesion structures, see arrows, Figure $2 \mathrm{C} 1$ ). The formation of such adhesion structures clearly contacting the pillar proves true adhesion of the keratinocytes to the pillar top. Moreover, areas of small keratinocyte islands formed on the FN-coated pillars (Figure 2D) parallel the situation found on conventional glass substrates (Figure 2B). The ability of IHGK to aggregate as such an island of interconnected cells is an important finding because the epithelial context in vivo is characterized by interconnected cells arranged in layers. The presence of cell-to-cell-interconnections visible on glass and the pillars as well (interconnections between cells are marked by arrows and seen in Figure 2B, glass; and Figure 2D, E FN-coated pillars) suggests that the biofunctionalized pillars arranged in $8 \mu \mathrm{m}$ distances render a tissue-adequate extracellular environment for studying tissue-specific cell functions, including early keratinocyte differentiation. This assumption is backed up by the finding that decreasing pillar distances localize one of the main epithelial-specific cellto-cell-contact forming desmosomal constituent desmoplakin, preferably at the cell periphery in islands where IHGKs have formed colonies of directly adjacent cells. This peripheral desmoplakin immunolocalization provides the basis for the formation of desmosome-based cell-to-cell contacts, rendering a further biological function in the context of epithelial homeostasis. This is best visible by comparison of pillars arranged in $14 \mu \mathrm{m}$ patterns (Figure 3A,A1) with those arranged in a $8 \mu \mathrm{m}$ distance (Figure $3 \mathrm{~B}, \mathrm{~B} 1$ ).

With respect to the pillars, we have observed failure in adhesion of IHGK when the pillar tops exceeded distances larger than $17 \mu \mathrm{m}$ (unpublished own observations). For this purpose, the maximum interspace used in this study was not larger than $14 \mu \mathrm{m}$.

Satisfactory cell viability on the biofunctionalized pillar "heads" is demonstrated in Figure 4A by the clear neutralred incorporation into the cell membranes in conjunction with the only faint trypan blue counterstain (Figure 4B). In addition, preliminary tests revealed an approximately $100 \%$ adhesion rate of the seeded cells (for description, see Supporting Information).

The effect of environmental changes, namely of micropillar interfaces with varying pillar distances on early keratinocyte (IHGK) differentiation, has been evaluated by IIF for K1. For defined distances, pillars arranged in arrays of 14, 11, and $8 \mu \mathrm{m}$ have been employed (Figure 5 see inlays). The green (merged with red as yellow) fluorescence signal of K1 showed a granular perinuclear pattern when IHGKs were seeded on large-distance pillar arrays (Figure 5A, $14 \mu \mathrm{m}$ ), while they exhibit a progressive cytoplasmic protein distribution with declining pillar distances (Figure 5B, $11 \mu \mathrm{m}$; Figure $5 \mathrm{C}$ and $\mathrm{D}, 8 \mu \mathrm{m})$. In this context, the most extensive cytoplasmic $\mathrm{K} 1$ distribution could be detected at the smallest pillar head distance of $8 \mu \mathrm{m}$ (Figure 5C and D). Concerning the keratin intermediate filaments, the normally seen cytoplasmic protein distribution starts from an organizing center associated with the nucleus. ${ }^{27}$ Thus, the cytoplasmic abundance of the keratin $\mathrm{K} 1$ protein can be considered as a biological correlate of the keratinocyte to fulfill its function in the epithelial context, which in our analysis is reflected by early differentiation. With respect to the IIF pattern found for $\mathrm{K} 1$ on the large-scale pattern of $14 \mu \mathrm{m}$, the perinuclear fluorescence may reflect an initial stage in K1 filament formation starting from the above-mentioned nucleus-associated organizing center. Therefore, the most extended cytoplasmic K1 distribution observed in large cell areas on the lowest scale pattern of $8 \mu \mathrm{m}$ may suggest the progressive formation of $\mathrm{K} 1$ intermediate filaments in the cytoplasm. In addition, this cytoplasmic K1 extension demonstrates that 

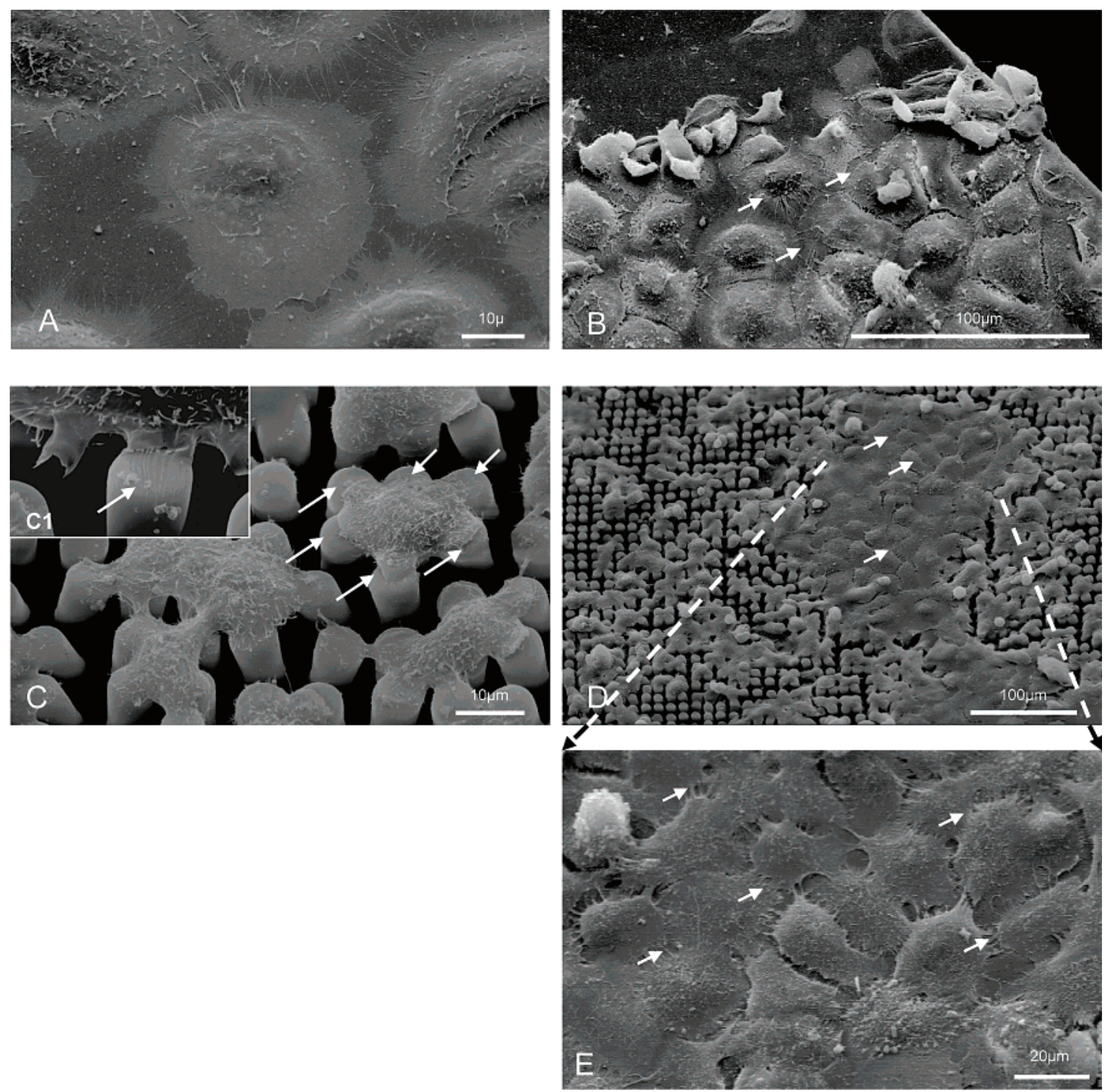

Figure 2. SEM pictures of IHGKs on glass or micropillar surfaces. (A) Single IHGKs on a glass slide exhibiting proper adhesion and cell-to-cell interconnections. (B) A nearly confluent IHGK layer on FN-coated glass surface, the cell-to-cell interconnections are marked by arrows, bar corresponds to $100 \mu \mathrm{m}$. (C) IHGKs on a FN-coated micropillar array, with pillar head interspaces of $8 \mu \mathrm{m}$, and a single keratinocyte covering seven pillars (see arrows), the inlay (C1) shows a high magnitude section of IHGK adhesion structures on a pillar head (see arrows), bar corresponds to $10 \mu \mathrm{m}$. (D) FN-coated micropillar array with a pillar head distance of $8 \mu \mathrm{m}$. The section shows IHKGs forming an island with cell-to-cell interconnections (see arrows), bar corresponds to $100 \mu \mathrm{m}$. For better visibility, Figure $2 \mathrm{E}$ provides picture section high-resolution magnification of cell-to-cell interconnections, bar corresponds to $20 \mu \mathrm{m}$.

the $8 \mu \mathrm{m}$ pillar pattern renders a favorable extracellular environment for early keratinocyte differentiation.

As already mentioned, $\mathrm{K} 1$ expression precedes that of K10 in the early differentiation program of epithelial keratinocytes. Interestingly, use of a K10-specific antibody, shown in Figure 6, demonstrates that K10 remains in a granular nucleus-associated pattern in IHGKs $24 \mathrm{~h}$ after seeding, irrespective of the pillar "head" distance (Figure 6A, $14 \mu \mathrm{m}$; Figure $6 \mathrm{~B}, 8 \mu \mathrm{m})$. This finding may contribute to the previously reported evidence in the delay of K10 appearance during the early keratinocyte maturation stages. ${ }^{9}$

To explore whether cytoplasmic extension observed for the early differentiation marker K1 in IHGK seeded on smallscale $8 \mu \mathrm{m}$ patterns implies a modulation of $\mathrm{K} 1$ on the transcriptional level, we have carried out semiquantitative (sqPCR) and quantitative RT-PCR (qPCR) experiments (meth- ods are described in detail in the Supporting Information). Hence, we performed RNA extraction of IHGKs after a cultivation period of $24 \mathrm{~h}$ from the same pillar arrays used for IIF. Using equal amounts of cDNA (1ng), sqPCR revealed increasing $\mathrm{K} 1 \mathrm{mRNA}$ transcript ratios associated with decreasing pillar head distances (Figure 7A). These data have been obtained by measuring gel band intensities (Figure 7A) of three independent experiments (methods are described in detail in the Supporting Information). In detail, pillar head distances of $14 \mu \mathrm{m}$ yielded the lowest K1 mRNA transcript level, compared with transcript levels obtained from IHGKs cultivated on decreasing pillar head distances, namely 11 and $8 \mu \mathrm{m}$ (Figure 7A). Cultivation of IHGKs on $8 \mu \mathrm{m}$ pillar distance revealed the highest K1 mRNA transcript ratio. Interestingly, the highest transcription ratio observed on arrays of $8 \mu \mathrm{m}$ coincided with the most extended cytoplasmic distribu- 

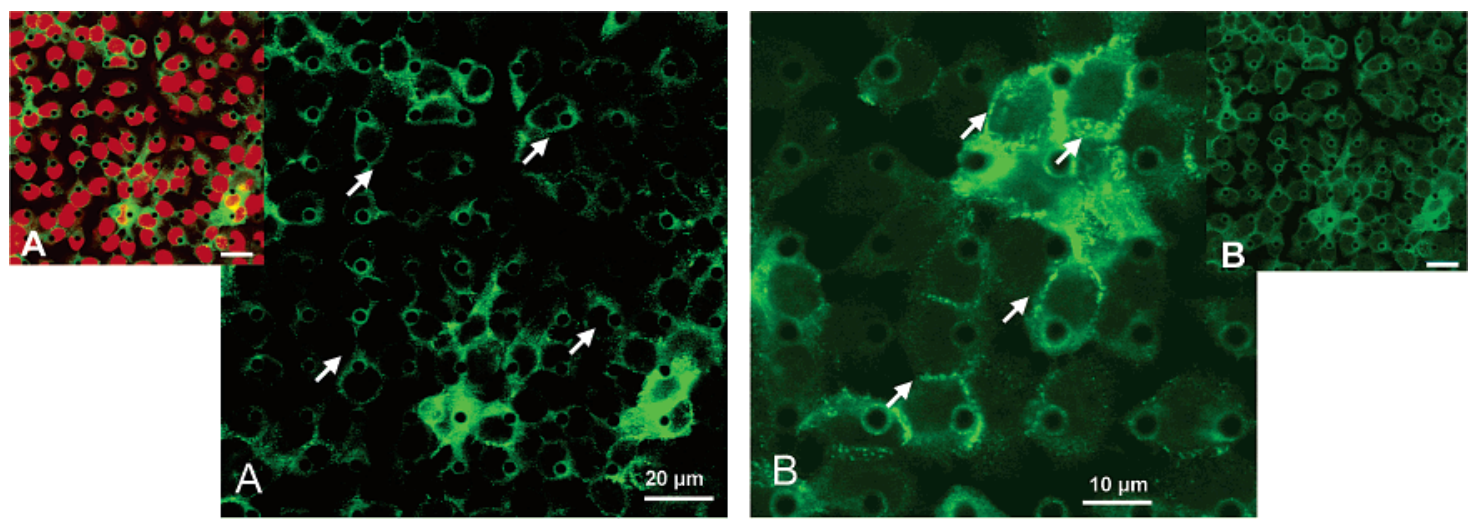

Figure 3. Indirect immunofluorescence (IIF) illustrates the desmosomal protein desmoplakin (DP) in IHGKs. (A) Weak, plasmamembraneassociated pattern (see arrows) for DP in IHGKs on a $14 \mu \mathrm{m}$ pillar array, bar corresponds to $20 \mu \mathrm{m}$. (A1) shows the same motif with cell nuclei resulting from the red propidium iodide (PI) counterstain, 63-fold magnification. (B) High-resolution picture shows the specific DP staining on an $8 \mu \mathrm{m}$ pillar array, illustrating the DP -localization at the cell periphery (see arrows), bar corresponds $10 \mu \mathrm{m}$. (B1) Overview of the same motif indicating the formation of IHGK islands (63-fold magnification).
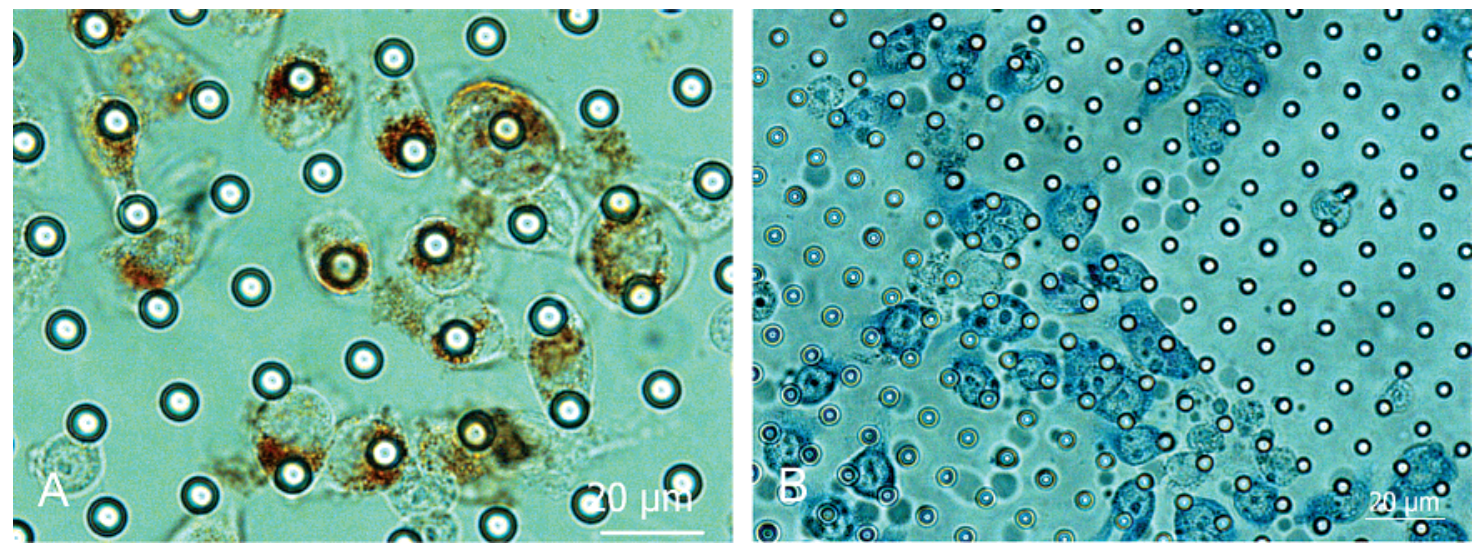

Figure 4. Light microscopy of IHGK viability analysis. (A) Cell membrane-bound neutral-red staining of IHGKs on pillars of $8 \mu \mathrm{m}$ pillar interspaces. The staining indicates living cells. (B) Faint trypan blue staining of IHGKs on pillar arrays of $8 \mu \mathrm{m}$ pillar interspaces, reinforcing cell viability. Bars correspond to $20 \mu \mathrm{m}$.

tion of the K1 protein. These coinciding findings strongly suggest that early keratinocyte differentiation is dependent on the distance of cell adhesion anchor points provided by the micropatterned biofunctionalized pillar surfaces. Moreover, they suggest that induction of early epithelial differentiation on both the transcriptional and the protein level as well may be modulated by the extracellular environment.

To specify our data obtained from sqPCR, we performed a qPCR experiment (methods are described in detail in the Supporting Information) to get quantitative information about the influence of different pillar head distances on IHGKs early cell differentiation. qPCR exhibits a comparable but even more pronounced increase in $\mathrm{K} 1$ expression levels with decreasing pillar head distances (Figure 7B). This is substantiated by the evidence that the K1 expression level of IHGKs, grown on an array of $11 \mu \mathrm{m}$ pillar distance, displayed a 2.7 fold higher expression level compared with the predefined control pillar distance of $14 \mu \mathrm{m}$ (Figure 7B). Moreover, at the pillar head distance of $8 \mu \mathrm{m}, \mathrm{K} 1$ expression level increased actually to a 4.5-fold higher expression compared with the control (Figure 7B).

In conclusion, our experiments using defined biofunctionalized microstructured interfaces demonstrate for the first time that the extracellular environment, independent from miscellaneous cell culture medium additives, modulates a biological cell function in human gingival keratinocytes (IHGKs) in a distance-dependent manner. Among the biological functions of epithelial keratinocytes, the pivotal process of early differentiation has been characterized in IHGKs by detection of a specific marker protein, reflected by keratin K1. Using these microarrays, declining pillar head distances proved a modulation of cytoplasmic K1 extension concomitant with increased K1 transcription levels. In coincidence with the findings obtained for K1 in IHGK, the peripheral desmoplakin immunolocalization observed on small distance pillar arrays may be a hint that other cell functions that contribute to epithelial tissue homeostasis are also addressed by the environmental conditions.

Thus, the biofunctionalized pillar structures open a new field to study the morphological and functional behavior of cells in relation to defined extracellular adhesion points. Therefore, distinct biological cell functions can be explored in a more in vivo-relevant context.

So far, the focus has been directed on naturally derived biomaterials that have proved effective in many basic clinical applications, but the need for custom-made matrices for 

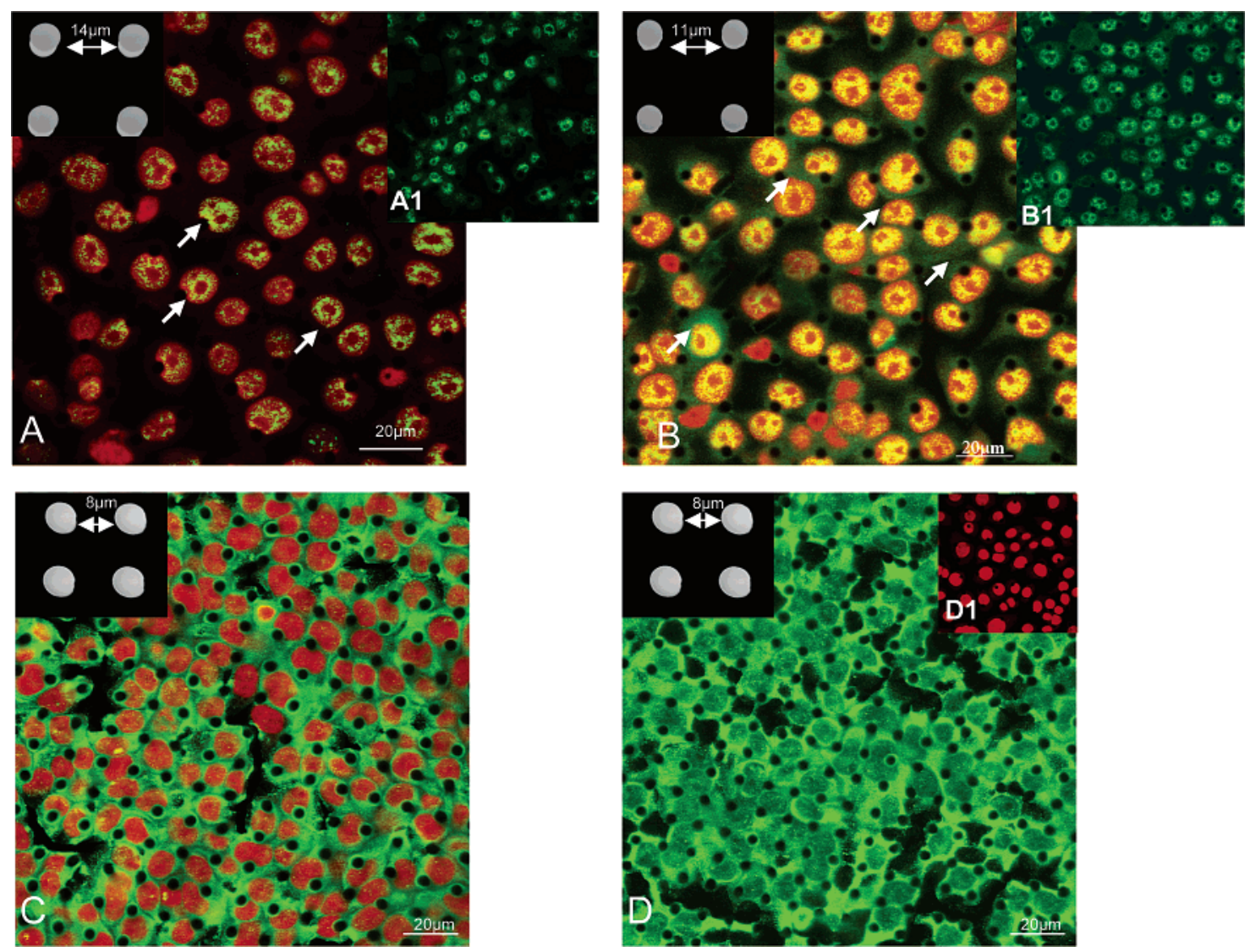

Figure 5. Indirect immunofluorescence illustrates the early differentiation marker K1 in IHGKs. (A) Granular, nucleus-associated pattern (see arrows) for K1 on a micropillar array of $14 \mu \mathrm{m}$ pillar distance, (A1) shows the same motif with the K1 green fluorescence signal only, to better discriminate the nucleus-associated pattern. (B) Increasing cytoplasmic K1 distribution on a micropillar array of $11 \mu \mathrm{m}$ pillar distance, (B1) indicating the K1 green fluorescence signal only, to better visualize the initiation of cytoplasmic K1 extension. (C) Most extensive cytoplasmic K1 distribution on the smallest pillar distance array of $8 \mu \mathrm{m}$, including propidium iodide (PI) nuclear counterstain, which is lacking in (D). Authenticity of cytoplasmic K1 extension is proven by the absence of green fluorescence following incubation of respective pillar array specimens with secondary fluorochrome-coupled antibody alone. Again, PI counterstain demarcates cell nuclei. Inlays illustrate SEM pictures to determine exact pillar distances. The scale bars correspond to $20 \mu \mathrm{m}$.
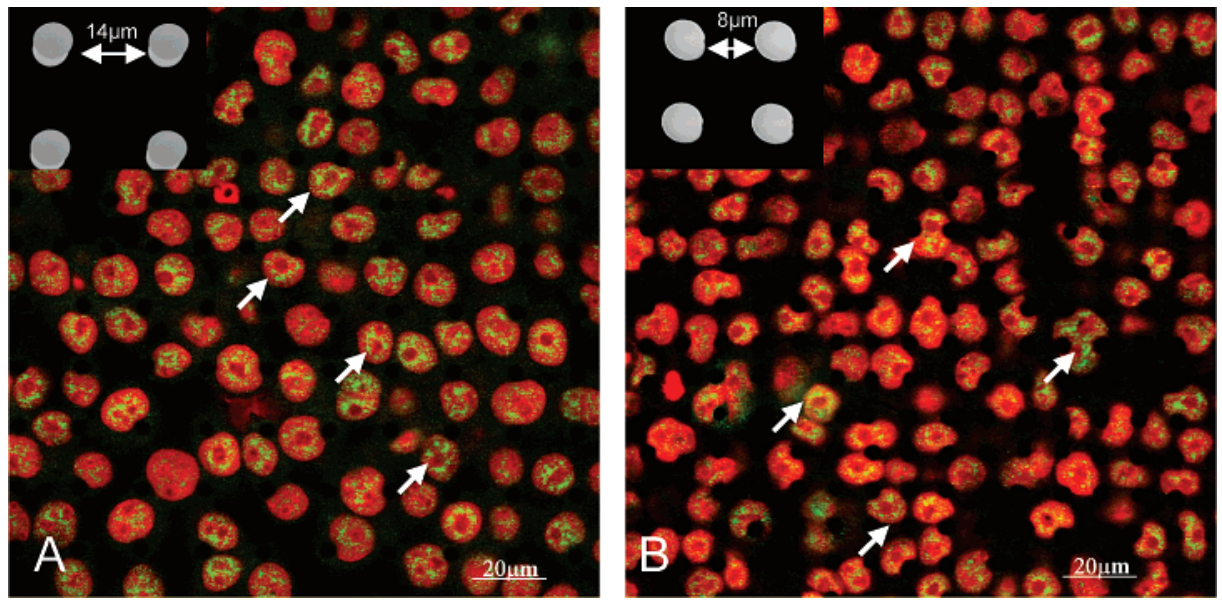

Figure 6. Indirect immunofluorescence (IIF) illustrates the intermediate-filament protein keratin 10 (K10) in IHGKs. (A) Granular, nucleusassociated pattern (see arrows) for K10 on a micropillar array of $14 \mu \mathrm{m}$ pillar distance, which appears similar on the $8 \mu \mathrm{m}$ pillar array (B, see arrows). Inlays illustrate SEM pictures to determine exact pillar distances. Bars correspond to $20 \mu \mathrm{m}$.

tissue-specific cell biological investigations has been raised in the past few years. These materials are still being developed to gain more control over the material and thus over the cellular behaviors they induce. ${ }^{29}$
Therefore, this study has addressed the field of building biomimetic elements, such as FN, into synthetic materials, in our case, PDMS. Consequently, the biophysical properties of extracellular matrix (ECM) components influence various 
A
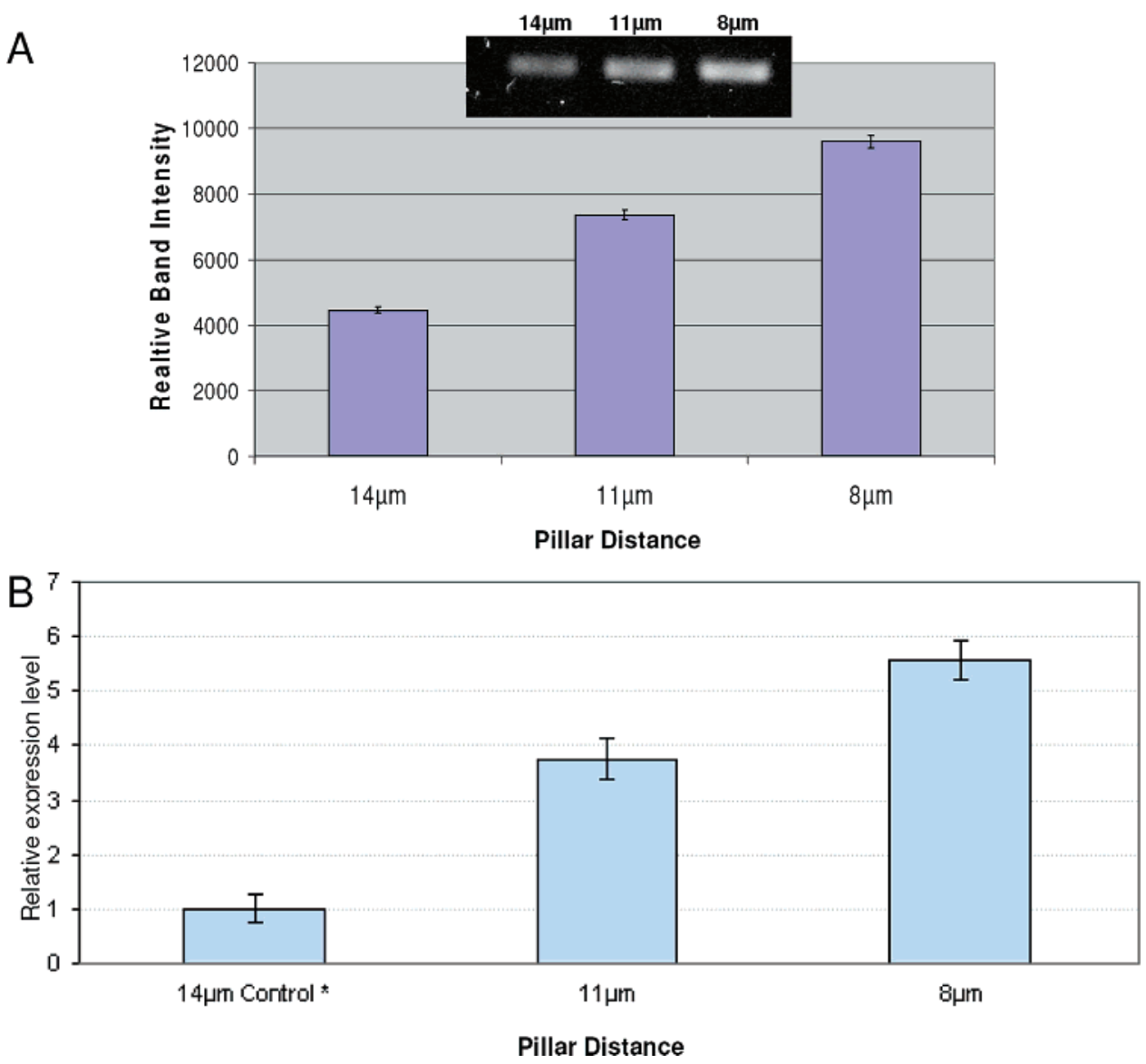

Figure 7. Expression profile of K1 detected by semiquantitative and quantitative RT-PCR. (A) K1 mRNA transcription of IHGKs analyzed on micropillar arrays with decreasing pillar head distances, 14,11 , and $8 \mu \mathrm{m}$, using equal amounts of cDNA. Measurements were carried out with image analysis software (ImageJ 1.37h, NIH, USA), determining different relative band intensities from RT-PCR products of three independent experiments. Mean \pm SEM, $n=3$. (B) The relative expression levels of K1 mRNA from IHGKs were analyzed using a modification of the $\Delta \Delta C_{\mathrm{T}}$ equation, which allows counting for differences in efficiencies $\left(E=10^{-1 / \text { slope }}\right)$ between the PCR reactions. ${ }^{28}$ The pillar distance of $14 \mu \mathrm{m}$ was predefined as control distance. The data were calculated using the software Gene Expression Macro provided with the iCycler. The data were normalized to the $C_{\mathrm{T}}$ of the unmodulated housekeeping gene (HKG) $\beta$-actin.

cell functions, including adhesion, differentiation, and migration. Moreover, the macromolecular structure of matrix components, such as FN, brings about modulation of important cell functions, including differentiation. ${ }^{30}$

A potential application for using biofunctionalized micropillar arrays is the study of mechanical forces in changing biological cell functions. Because of the tight connection between the cytoskeleton and the ECM through cell surface receptors, cells sense and respond to the mechanical properties of their environment by converting mechanical signals into chemical signals. ${ }^{31,32}$ Therefore, a further advantage of the micropillar arrays is that they allow for studying defined mechanical forces directed on single cells by micromanipulating single pillar heads and investigating forces which cells exert on their adjacent extracellular environment. To allow for a first approximation of the forces directed from the pillars to the IHGKs used in this study, we calculated the spring constant of FN-coated pillars distanced $8 \mu \mathrm{m}$ to each other to be $0.17 \mathrm{~N} / \mathrm{m}$ (pillar dimension: $5 \mu \mathrm{m}$ diameter, height $15 \mu \mathrm{m}$; for details see Supporting Information). Moreover, Figure 8 shows for the first time the pillar deflection of single epithelial keratinocytes growing on the above-mentioned $8 \mu \mathrm{m}$ pattern.

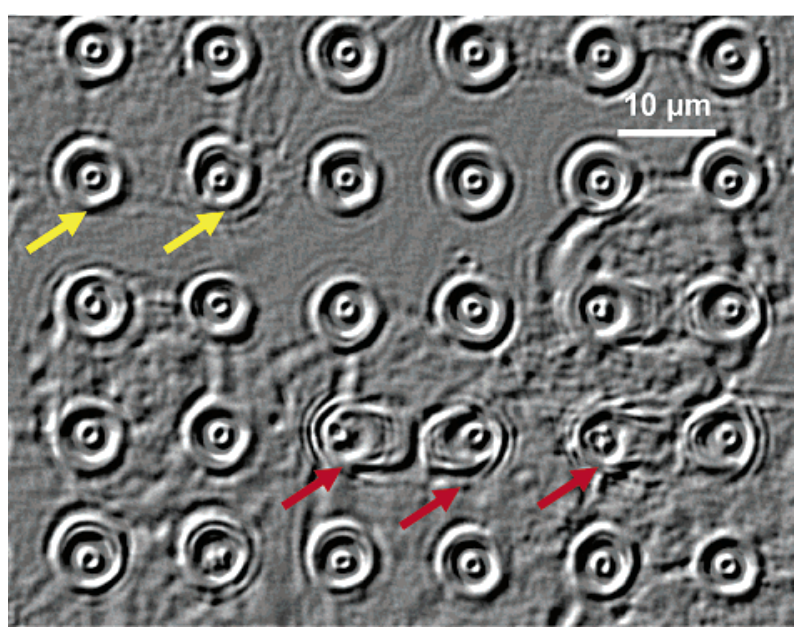

Figure 8. Bright-field live cell image of a micropillar array with $8 \mu \mathrm{m}$ pillar interspaces depicting single IHGKs on FN-coated pillar tops either with (red arrows) or without deflection (yellow arrows). Bar corresponds to $10 \mu \mathrm{m}$.

Transformation of bright-field live cell images of deflected and nondeflected control pillars into a coordinate field allowed for calculation of cell-emanating traction forces, which could be determined to be in a range from 30-110 
$\mathrm{nN} / \mu \mathrm{m}^{2}$. Therefore, the features of PDMS pillar arrays make them a valuable tool to explore biological cell functions of cells derived from solid tissues in a defined topologically structured biomimetic environment. For the future, evidence obtained from biofunctionalized elastic micropillar arrays may help to understand the requirements of cells from different tissues with respect to a biomaterial for optimizing tissue regeneration or tissue integration of implants.

Acknowledgment. The Dietmar-Hopp-Stiftung, GmbH, St. Leon-Rot, Förderbereich Medizin is acknowledged for financial support (grant 12/2005 to Thorsten Steinberg and Pascal Tomakidi). Additional financial support has been supplied by the Deutsche Gesellschaft für Zahn-, Mund- and Kieferheilkunde (DGZMK) to Eva Muessig (grant 11/2006). The work has also been initiated in the "BIOQUANT" Research Network of the University of Heidelberg, Germany.

Supporting Information Available: Experimental details including pillar fabrication, cell culture protocol for IHGKs, indirect immunofluorescence, and details to the methods for semiquantitative and quantitative RT-PCR. This material is available free of charge via the Internet at http://pubs.acs.org.

\section{References}

(1) Arnold, M.; Cavalcanti-Adam, E. A.; Ulmer, J.; Blummel, J.; Eck, W.; Kantlehner, M.; Kessler, H.; Spatz, J. P. ChemPhysChem 2004, 19, 383-388.

(2) Roos, W.; Ulmer, J.; Grater, S.; Surrey, T.; Spatz, J. P. Nano Lett. 2005, 5, 2630-2634.

(3) Tan, J. L.; Tien, J.; Pirone, D. M.; Gray, D. S.; Bhadriraju, K.; Chen, C. S. Proc. Natl. Acad. Sci. U.S.A. 2003, 100, 1484-1489.

(4) du Roure, O.; Saez, A.; Buguin, A.; Austin, R. H.; Chavrier, P.; Silberzan, P.; Ladoux, B. Proc. Natl. Acad. Sci. U.S.A. 2005, 102, 2390-2395.

(5) Stark, H. J.; Baur, M.; Breitkreutz, D.; Mirancea, N.; Fusenig, N. E. J. Invest. Dermatol. 1999, 112, 681-691.

(6) Rice, R. H.; Green, H. Cell 1979, 18, 681-694.
(7) Dale, B. A. Am. J. Dermatopathol. 1985, 7, 65-68.

(8) Green, H.; Fuchs, E.; Watt, F. Cold Spring Harbor Symp. Quant. Biol. 1982, 46 (Part 1), 293-301.

(9) Lu, B.; Rothnagel, J. A.; Longley, M. A.; Tsai, S. Y.; Roop, D. R. J. Biol. Chem. 1994, 269, 7443-7449.

(10) Gutman, A.; Kornblihtt, A. R. Proc. Natl. Acad. Sci. U.S.A. 1987, 84, 7179-7182.

(11) Clark, R. A.; Folkvord, J. M.; Wertz, R. L. J. Invest. Dermatol. 1985, 84, 378-383.

(12) Hynes, R. O.; Yamada, K. M. J. Cell Biol. 1982, 95, 369-377.

(13) Clark, R. A.; Lanigan, J. M.; DellaPelle, P.; Manseau, E.; Dvorak, H. F.; Colvin, R. B. J. Invest. Dermatol. 1982, 79, 264-269.

(14) Watt, F. M.; Kubler, M. D.; Hotchin, N. A.; Nicholson, L. J.; Adams, J. C. J. Cell Sci. 1993, 106 (Part 1), 175-182.

(15) Narayanan, A. S.; Clagett, J. A.; Page, R. C. J. Dent. Res. 1985, 64, 1111-1116.

(16) Hennings, H.; Holbrook, K.; Steinert, P.; Yuspa, S. Curr. Probl. Dermatol. 1980, 10, 3-25.

(17) Roop, D. R.; Huitfeldt, H.; Kilkenny, A.; Yuspa, S. H. Differentiation 1987, 35, 143-150.

(18) Menon, G. K.; Grayson, S.; Elias, P. M. J. Invest. Dermatol. 1985, 84, 508-512.

(19) Feghali-Assaly, M.; Sawaf, M. H.; Serres, G.; Forest, N.; Ouhayoun, J. P. J. Periodontal Res. 1994, 29, 185-195.

(20) Roos, W.; Ulmer, J.; Grater, S.; Surrey, T.; Spatz, J. P. Nano. Lett. 2005, 5, 2630-2634.

(21) O’Keefe, E. J.; Payne, R. E., Jr.; Russell, N.; Woodley, D. T. J. Invest. Dermatol. 1985, 85, 125-130.

(22) Yamada, Y.; Kleinman, H. K. Curr. Opin. Cell Biol. 1992, 4, 819823.

(23) Ganz, A.; Lambert, M.; Saez, A.; Silberzan, P.; Buguin, A.; Mege, R. M.; Ladoux, B. Biol. Cell 2006, 98, 721-730.

(24) Molina, T.; Kabsch, K.; Alonso, A.; Kohl, A.; Komposch, G.; Tomakidi, P. J. Dent. Res. 2001, 80, 1984-1889.

(25) Patankar, N. A. Langmuir 2004, 20, 8209-8213.

(26) Gumbiner, B. M. Cell 1996, 84, 345-357.

(27) Eckert, B. S.; Daley, R. A.; Parysek, L. M. J. Cell Biol. 1982, 92, 575-578.

(28) Livak, K. J.; Schmittgen, T. D. Methods 2001, 25, 402-408

(29) Lutolf, M. P.; Hubbell, J. A. Nat. Biotechnol. 2005, 23, 47-55.

(30) Garcia, A. J.; Reyes, C. D. J. Dent. Res. 2005, 84, 407-413.

(31) Galbraith, C. G.; Skalak, R.; Chien, S. Cell Motil. Cytoskeleton 1998, 40, 317-330.

(32) Geiger, B.; Bershadsky, A. Curr. Opin. Cell Biol. 2001, 13, 584-592.

NL062271Z 